\title{
EchoGéo
}

$44 \mid 2018$

Les valorisations territoriales et touristiques du street art

\section{Les espaces du graffiti dans les capitales touristiques : l'exemple de Paris et Berlin}

Julie Vaslin

\section{OpenEdition}

Journals

Electronic version

URL: https://journals.openedition.org/echogeo/15348

DOI: 10.4000/echogeo. 15348

ISSN: 1963-1197

Publisher

Pôle de recherche pour l'organisation et la diffusion de l'information géographique (CNRS UMR 8586)

Electronic reference

Julie Vaslin, "Les espaces du graffiti dans les capitales touristiques : l'exemple de Paris et Berlin", EchoGéo [Online], 44 | 2018, Online since 31 July 2018, connection on 31 July 2021. URL: http:// journals.openedition.org/echogeo/15348 ; DOl: https://doi.org/10.4000/echogeo.15348

This text was automatically generated on 31 July 2021.

EchoGéo est mis à disposition selon les termes de la licence Creative Commons Attribution - Pas d'Utilisation Commerciale - Pas de Modification 4.0 International (CC BY-NC-ND) 


\title{
Les espaces du graffiti dans les capitales touristiques : l'exemple de Paris et Berlin
}

\author{
Julie Vaslin
}

\section{Introduction}

«Paris se laisse devancer par les grandes villes voisines (Berlin, Amsterdam, Londres, Barcelone...), qui ont su structurer leur offre et leur image pour attirer cette clientèle » (Mairie de Paris, 2017, p. 8).

1 Attirer les "city breakers ", ces jeunes touristes qui parcourent pour un weekend ou quelques jours les grandes villes européennes (Guérin, 2015), tel est l'un des objectifs du schéma de développement touristique de la Mairie de Paris et du Grand Paris, à l'aube des années 2020. Parmi les villes «concurrentes" identifiées par les acteurs du tourisme parisien la capitale allemande, Berlin, est considérée comme un véritable " exemple à suivre " en matière de renouvellement des formes de tourisme urbain (Béal, 2014). Au cœur de la notoriété alternative de Berlin, le street art apparaît comme la figure de proue du dynamisme culturel et touristique de la métropole allemande. Par exemple, les photographes fondateurs du Fresh Street Art Tour, un tour parisien de découverte du street art, estiment que "Paris n'est pas assez dynamique et les gens disent "la Tour Eiffel est là et si je reviens dans 10 ans, la Tour Eiffel sera toujours là". Et du coup, ils vont se tourner vers des villes qui ont une image plus dynamique, [comme] Berlin, Lisbonne, des choses comme ça »1.

2 En opposant le haut-lieu touristique qu'est la Tour Eiffel au street art berlinois, ce guide parisien réactualise l'opposition canonique entre l'image patrimoniale intemporelle de Paris et l'image culturelle alternative de Berlin, faisant du street art le symbole d'une offre touristique «hors des sentiers battus » réussie (Gravari-Barbas et Delaplace, 2015). 
3 Cette opposition peut cependant être nuancée. À Berlin comme à Paris, de nombreuses peintures murales sont effacées et leurs auteurs sont régulièrement poursuivis par les autorités publiques. À Paris comme à Berlin, la promotion culturelle de certaines peintures murales et de leurs auteurs a pourtant lieu dans des contextes particuliers. En somme, on assiste dans les deux villes à un tri des graffitis ${ }^{2}$, qui s'opère à travers des dispositifs de répression et promotion de ces peintures, comparables d'une ville à l'autre.

4 Largement documentés par des sociologues anglo-saxons (Ferrell, 1996 ; Austin, 2001), les enjeux de la répression des graffitis ont été récemment mis en perspective avec ceux de leur promotion officielle, dans les travaux de Ronald Kramer. Dans une perspective néo-marxiste, R. Kramer propose de comprendre les politiques newyorkaises à l'aune des intérêts économiques qu'elles contribuent à défendre. Il soutient l'idée que les autorités new-yorkaises, qu'elles effacent le graffiti dans la rue ou le promeuvent en galerie, sont animées par l'ambition d'« extraire le profit maximum des usages du paysage urbain» (Kramer, 2016). Avant lui, le géographe Tim Cresswell constatait déjà ironiquement que l'image choisie par le parc d'attraction Disney world pour représenter la ville de New York est un train couvert de graffitis. Analysant dans une perspective plus foucaldienne la politique new-yorkaise d'effacement des graffitis, il défend l'idée que le maire n'est pas tant préoccupé par la préservation des bâtiments de New York, que par la préservation de « New York comme un symbole du contrôle, de l'ordre et de l'harmonie » (Cresswell, 1996, p. 46).

Dans le prolongement de ces travaux, on s'intéresse ici à la manière dont les acteurs urbains parisiens et berlinois gouvernent le graffiti pour produire des espaces publics attractifs et touristiques. Pour étudier l'administration du graffiti, on propose une analyse du gouvernement de l'espace public (Fleury, 2007), et plus particulièrement de son esthétisation (Boichot et Guinard, 2013), à la croisée entre les politiques de propreté et les politiques culturelles. Précisément, cet article prend appui sur une enquête qualitative et comparée de sociologie de l'action publique (Hassenteufel, 2008 ; Lascoumes et Le Galès, 2012) composée d'archives, d'entretiens, d'observations et de photographies, réalisée à Paris et Berlin entre 2011 et 2017 (Vaslin, 2017). Essentiellement focalisée sur les acteurs du gouvernement urbain (Payre, 2008), cette enquête nous permet de déconstruire les représentations associées aux images légitimes $d u$ «bel espace public » pour celles et ceux qui gouvernent la ville, en fonction des espaces. Loin d'être passifs dans ce processus, les auteurs de graffitis sont en interactions permanentes avec les autorités urbaines et il s'agit moins ici de documenter la nature de ces interactions, que de questionner les étiquetages dont leurs productions font l'objet, en fonction des espaces où elles se déploient. Autrement dit, il s'agit dans cet article de révéler le processus par lequel les autorités urbaines soit assimilent les graffitis à des souillures dans les centralités touristiques, soit les tolèrent voire les promeuvent en tant qu'art sous le terme de street art ${ }^{3}$ dans d'autres espaces urbains, plus périphériques ou marginaux.

6 La perspective comparée de notre enquête permet enfin de repérer les logiques convergentes qui président à la régulation des graffitis dans les espaces urbains, dans des villes aux morphologies et aux histoires culturelles pourtant contrastées. Ainsi, si Berlin accueille visiblement davantage de graffitis dans son espace public que Paris, on constate l'existence d'une même logique de différenciation des esthétiques urbaines 
entre quartiers, favorisant la constitution d'une offre touristique variée à l'échelle de la ville, mais paradoxalement de plus en plus standardisée d'une métropole à l'autre.

7 Nous montrerons que les traitements spatialement différenciés du graffiti dans ces deux capitales sont comparables puisque (1) bien qu'esthétiquement contrastées, les centralités urbaines parisiennes et berlinoises sont des espaces dénués de tout graffiti, (2) et que c'est dans des espaces périphériques que la mise en tourisme du «street art» s'opère, (3) quitte à favoriser la standardisation des esthétiques, dans un contexte général de développement concurrentiel du tourisme hors des sentiers battus.

\section{Paris, Berlin : des centralités touristiques « propres »}

Berlin, " pauvre mais sexy » ${ }^{4}$ et Paris, « ville lumière », ont acquis au fil de leur histoire deux esthétiques urbaines très contrastées. Espaces touristiques, les " hauts-lieux $»^{5}$ de ces deux capitales sont néanmoins entretenus selon des logiques convergentes de gouvernement, promouvant la mise à l'écart de toute forme d'esthétique concurrente graffitis en tête.

«Alors que Paris offre le visage d'une ville largement patrimonialisée, les bombardements alliés puis la reconstruction ont fait disparaître à Berlin une grande partie des quartiers historiques » (Fleury, 2014, p. 225).

En quelques mots, le géographe Antoine Fleury résume ainsi le contraste esthétique que l'on peut observer entre les espaces publics de deux capitales, façonnés par leurs histoires respectives. La capitale allemande connaît au $\mathrm{XX}^{\mathrm{e}}$ siècle une histoire segmentée qui s'inscrit dans les évolutions rapides et nombreuses de son paysage. Devenue capitale lors de l'unification de l'Allemagne en 1871 et scène d'une révolution socialiste en 1918, elle est la capitale de la République de Weimar dans les années 1920, puis celle du régime nazi dans les années 1930 et 1940. Occupée par quatre pays après la Seconde Guerre mondiale, elle est divisée en deux par un mur symbole de la Guerre Froide durant quarante ans et ce n'est qu'en 1999 qu'elle redevient la capitale d'une Allemagne réunifiée. Chacune de ces époques a marqué l'espace public berlinois au gré de démolitions et reconstructions successives. Au début des années 2000, Berlin devient progressivement une destination touristique, construite d'abord autour de hauts-lieux symboliques de son histoire politique (Sanson, 2014; Bocquet et Laborier, 2016). La capitale française, quant à elle, est une métropole culturelle européenne majeure depuis le XVII ${ }^{\mathrm{e}}$ siècle. Construite à la fin du XIX siècle, l'homogénéité haussmannienne du centre-ville est parsemée de nombreux monuments incarnant les centralités politiques et culturelles du pays. Aux abords des rives de Seine, le bâti et les espaces publics du cœur historique de Paris sont classés au patrimoine mondial de l'Unesco depuis 1991 (Marchand, 1993). Ville la plus visitée d'Europe, Paris et sa région ont comptabilisé en 2015 près de 75 millions de nuitées touristiques, contre environ 30 millions pour Berlin, classée au vingtième rang du classement des espaces touristiques européens ${ }^{6}$. Il est cependant important de noter qu' "entre 2005 et 2015, [...] le taux de croissance le plus élevé [du nombre total de nuitées passées par des touristes internationaux] a été constaté à Berlin (10,5\% par an) $»^{7}$.

Dans ces contextes urbains marqués par des histoires différentes, le développement du tourisme est une réalité contemporaine partagée. À Paris comme à Berlin, les hautslieux de la ville font l'objet d'attentions particulières en matière de maintien de l'ordre, 
social et esthétique, au nom de la fréquentation massive dont ils font l'objet. Et malgré les nombreuses différences qui marquent la structuration de la lutte anti-graffiti à Paris et à Berlin (voir encadré), les graffitis ont d'abord été perçus unanimement par les acteurs urbains comme autant de désordres venus perturber l'image de marque de ces hauts-lieux.

\section{Encadré - Les cadres politiques de la lutte anti-graffiti à Paris et à Berlin}

D'un point de vue juridique, en France dans le Code Pénal comme en Allemagne dans le Strafgesetzbuch, les graffitis sont d'abord qualifiés comme des « dommages aux biens ». Depuis 1994, différents groupes d'investigation se sont succédés au sein de la police berlinoise pour poursuivre les auteurs de graffitis ayant commis un dommage aux biens. Le Landeskriminalamt 264 mène aujourd'hui, à l'échelle berlinoise, une politique de prévention et de répression contre les auteurs de toute forme de graffiti réalisée sans l'autorisation du propriétaire du mur. Ne disposant pas de pouvoirs de police, la Mairie de Paris n'a pas développé de dispositif de répression à l'encontre des auteurs de graffitis (il en existe à l'échelle nationale). En revanche, l'arrêté municipal du 15 octobre 1999 prévoit la prise en charge par la Ville de l'effacement systématique de tous les graffitis dans un délai de quelques jours. Renforcé, ce dispositif implique aujourd'hui des entreprises prestataires, chargées de repérer et effacer quotidiennement tous les graffitis de Paris, sauf en cas d'avis contraire explicite du propriétaire des murs. À Berlin, l'entreprise parapublique de propreté, la Berliner StrassenReinigung (BSR), ne se préoccupe pas, en théorie, de l'effacement des graffitis. Cependant, la responsable de la propreté dans l'arrondissement de Mitte affirmait en entretien que les graffitis identifiés par ses agents dans les hauts-lieux touristiques de la ville étaient rapidement effacés par ses services. L'effacement des graffitis sur les bâtiments publics est parallèlement assuré par les Ordnungsämter (bureaux de l'ordre) des Arrondissements berlinois.

11 Au début des années 1990, à Paris, la lutte anti-graffiti s'intensifie dans les faits comme dans les discours: "Les graffitis prolifèrent sur les murs de la Capitale. Ils ne constituent pas seulement une agression visuelle mais [...] ils dégradent environnement et patrimoine $»^{8}$.

12 En 1999, le maire de Paris, Jean Tibéri, propose le vote d'un arrêté municipal prévoyant le repérage et l'effacement systématique de tous les graffitis de la ville, par les acteurs municipaux: "Sus aux graffitis! Afin de lutter contre les graffitis qui portent gravement atteinte aux immeubles mais aussi au paysage de Paris, Jean Tibéri vient de mettre en place un enlèvement systématique et gratuit $»^{9}$.

Pris dans les politiques de propreté, ce dispositif d'effacement est accompagné à Paris par un entretien comparativement plus fort des espaces centraux de la ville. Bien que moins peuplés, ces espaces accueillent l'essentiel des millions de touristes qui participent à un renouvellement des souillures plus rapide et considéré comme plus urgent. Mais l'entretien des rues ne se limite pas à une ambition technique, il véhicule également une esthétique du «propre» commune aux centres villes, dont les graffitis sont généralement bannis.

De la même manière, à Berlin, la lutte anti-graffiti n'est pas seulement formulée en termes de dommages aux biens. Lors de l'inauguration d'un congrès international antigraffiti en 2006, le maire de Berlin affirme clairement l'articulation des enjeux de protection de la propriété à ceux de la préservation de l'image de la ville pour justifier la lutte anti-graffiti : "The material damage caused bu graffiti to private buidings is immense. [...] An increasing number of people see the work of comparatively small 
group of taggers as a disfigurement of public space and a public nuisance. At the invitation of the Berlin-based association "Noffiti », experts [...] will be meeting in Berlin for the second time to exchange experience, to talk about strategies for preserving the cityscape, and to discuss the careful use of public spaces » (Wowereit, 2006, p. 9).

La priorité donnée aux "vitrines" touristiques dans les politiques de propreté s'exprime dans les moyens techniques et humains mobilisés pour l'entretien des axes les plus fréquentés dans les centres de Paris et de Berlin, supérieurs à ceux utilisés pour entretenir les espaces résidentiels. En effet, que l'effacement des graffitis soit assuré par des entreprises délégataires de service public (Paris) ou sous l'effet de commandes des propriétaires d'immeubles (Berlin), on observe une très faible présence de ces peintures sur les façades des quartiers touristiques centraux et plus aisés.

\section{Illustration 1 - Les murs immaculés du $7^{\mathrm{e}}$ arrondissement aux alentours de la Tour Eiffel, à Paris}

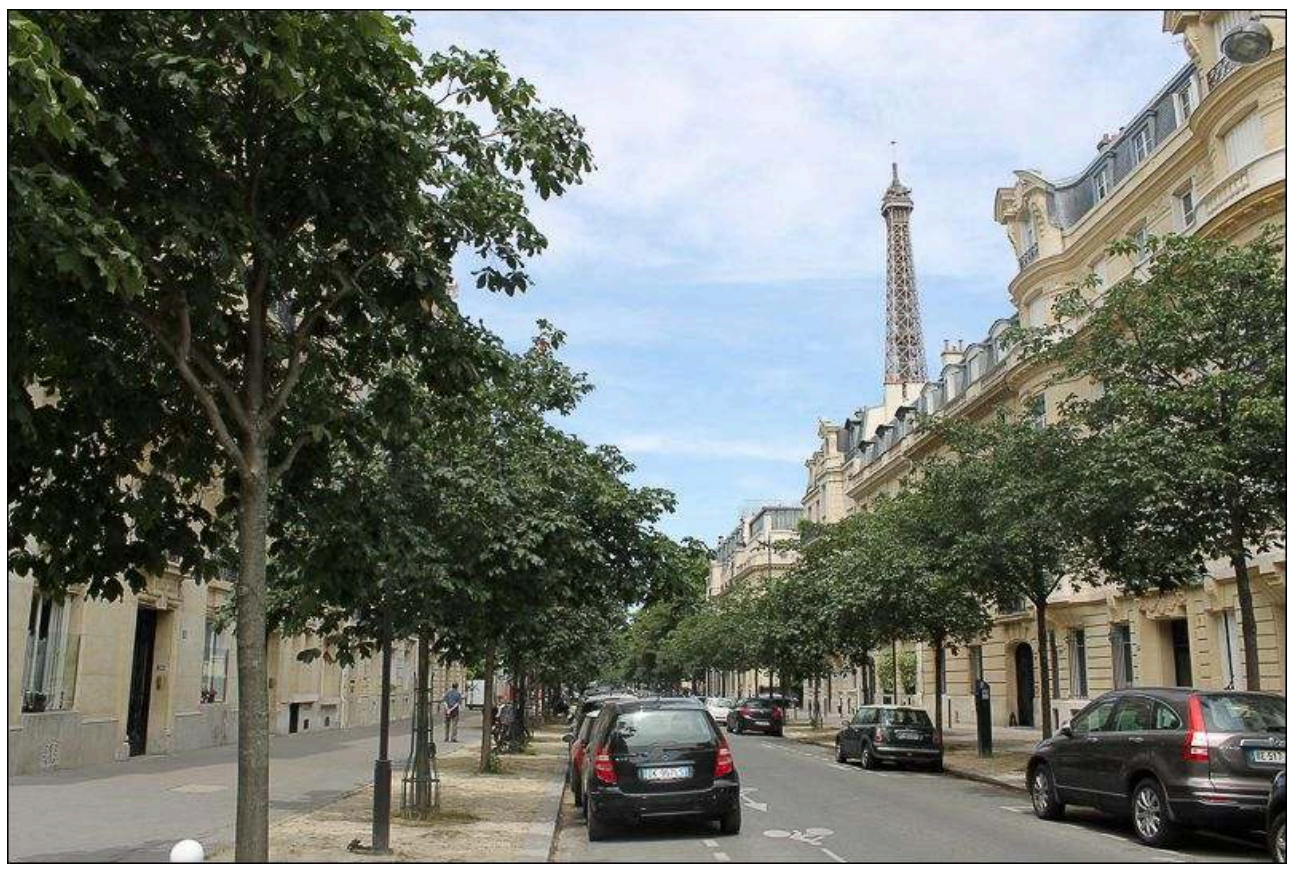

Auteur : J. Vaslin, 2014. 
Illustration 2 - Les murs immaculés de Mitte, aux alentours du Hackescher Markt, à Berlin

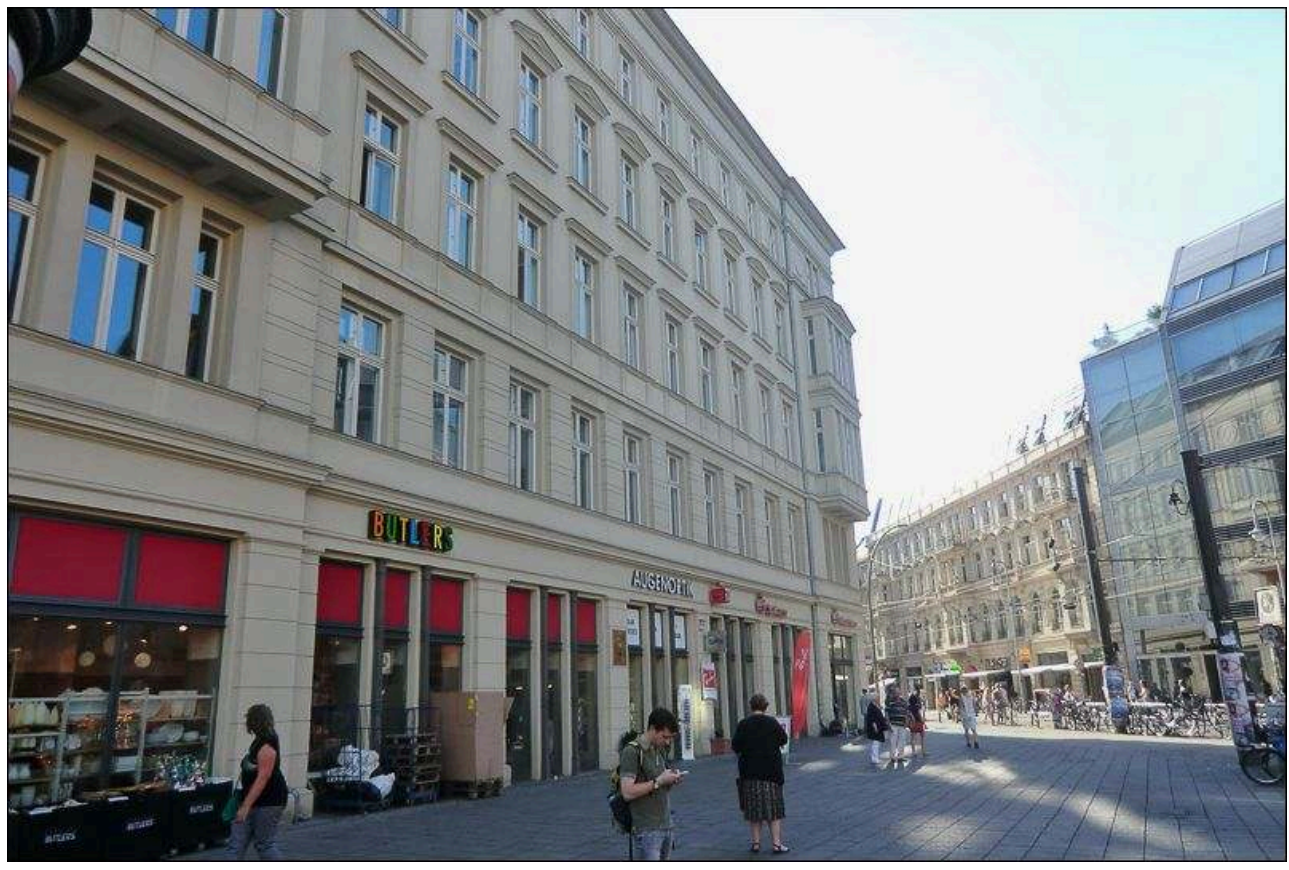

Auteur: J. Vaslin, 2017.

16 L'absence sensible de graffitis nous amène à faire l'hypothèse que les habitants propriétaires de leurs logements de centre-ville seraient davantage disposés à mandater des entreprises prestataires pour entretenir leurs façades, que les locataires des logements des quartiers populaires de la ville. Ayant fait le même constat dans les rues de Paris (Vaslin, 2017), la sociologie urbaine nous permet ici de comprendre le rapport différencié qu'entretiennent les habitants à l'esthétique de leur quartier de résidence (Pinçon et Pinçon-Charlot, 1989). Marqués par le patrimoine historique ou la centralité géographique, ces espaces peuvent parfois servir de décor à un haut-lieu touristique et, à ce titre, bénéficier d'interventions renforcées des services de propreté. Dans ce contexte, peu de graffitis spontanés et illégaux sont amenés à perdurer sur les murs des centres villes.

Enfin, les hauts-lieux touristiques, ne sont pas nécessairement cantonnés aux espaces centraux de la ville. À Berlin, l'East Side Gallery, située dans l'arrondissement Friedrichshain, au sud-est de la ville, est un vestige du mur de Berlin recouvert de fresques réalisées spontanément par des artistes internationaux au début des années 1990. Lors de sa rénovation en 2009, les mêmes artistes sont venus peindre les mêmes fresques, elles-mêmes recouvertes d'un vernis anti-graffiti afin de "repeindre le tout et d'empêcher de nouvelles souillures ", faisant de l'East Side Gallery une œuvre " protégée [avec un vernis] qui permet de nettoyer sans faire couler l'œuvre ${ }^{10}$.

Même si les moyens mis en œuvre pour atteindre cet objectif diffèrent, à Paris comme à Berlin, les graffitis sont gouvernés dans les espaces touristiques centraux comme des souillures. La propreté de ces espaces, telle qu'elle est définie par les acteurs urbains, participe d'un ordre esthétique constamment entretenu, dans lequel les graffitis apparaissent comme un désordre, quelles que soient leurs formes. L'effacement des graffitis fait donc partie de la production de l'image de marque des centres villes. Il est cependant difficile pour les acteurs urbains de maintenir cet état de «blancheur » sur 
toutes les façades de la ville. C'est donc à défaut de pouvoir assurer le maintien d'un même ordre esthétique et de propreté sur tout le territoire d'une ville que les autorités urbaines, publiques et privées, tolèrent le développement des expressions murales spontanées, dans les espaces publics situés à la périphérie des hauts-lieux touristiques.

\section{La mise en tourisme des graffitis dans les marges métropolitaines}

19 Le développement des graffitis et leur légitimation progressive au rang d'objets culturels et touristiques s'opère dans des espaces en marge des centralités touristiques des villes. Ces lieux ont pour point commun d'accueillir une activité culturelle alternative intense et de faire l'objet de politiques d'effacement des graffitis moins fortes. Présents dans différents types de marges urbaines, les graffitis sont parfois tolérés par les autorités, voire promus au rang d'emblèmes territoriaux, dans certains quartiers périphériques. Ce processus de légitimation implique une sélection des formes de graffitis promus et de leurs auteurs, ainsi qu'une redéfinition institutionnelle de la pratique, sous le label street art. Si quelques travaux font état de la transformation des carrières des auteurs de graffitis (Lemoine, 2012; Weill, 2014), de "déviante » à " artistique", notre propos se concentre ici sur l'évolution des conditions politiques dans lesquelles les auteurs de graffiti ont accès à l'espace public. Autrement dit, il s'agit moins de questionner la manière dont les auteurs se positionnent face à la "marge » que de comprendre comment les frontières de cette marge évoluent sous l'effet des politiques publiques.

À Paris, quelques plasticiens décident à la fin des années 1970 d'exercer leur art in situ, dans l'espace urbain. Diplômés des Beaux-Arts, membres de collectifs ou artistes indépendants, ils utilisent le pochoir pour marquer de leurs peintures les murs des $20^{\mathrm{e}}$ et $13^{\mathrm{e}}$ arrondissements, quartiers populaires situés à la périphérie du centre (quartiers de Belleville, Ménilmontant et la Butte aux Cailles en tête). Les années 1980 sont ensuite le décor de l'importation du writing américain ${ }^{11}$. Pour s'exercer, les premiers graffeurs parisiens peignent leurs noms (sous formes de signatures simples, les tags, ou complexes, les graffs) dans différentes marges urbaines : des palissades qui entourent le chantier de la pyramide du Louvre aux terrains vagues situés en périphérie, les lieux qui échappent aux politiques d'effacement sont prisés par les auteurs de graffitis. Le tournant des années 1990 est enfin marqué par la massification de la pratique du writing dans les souterrains, du métro aux catacombes. Palissades, terrains vagues, souterrains : ces espaces sont des « marges » de la ville en ceci qu'ils sont «marqués du sceau de l'abandon, de l'indéfini, de l'inachevé, du laid, de la souillure, de la saleté, de l'inculture ", ils sont «situés dans la ville [mais] symbolisent cependant le dehors de la ville, ce qu'elle cherche à cacher » (Grésillon, 2008). Ces marges constituent par ailleurs des espaces de sociabilité essentiels pour les auteurs de graffitis qui, en leur sein, se rencontrent, s'exercent, développent des styles esthétiques variés. À Berlin, l'ouest de la ville est marqué depuis les années 1970 par une intense activité politique contestataire, le quartier de Kreuzberg ayant en particulier attiré de nombreux objecteurs de conscience. Les mouvements écologistes, féministes et libertaires qui se développent à Berlin Ouest font du graffiti un outil central de leur répertoire d'action collective (Tilly, 1984) et s'en emparent pour marquer le paysage urbain. À la fin des années 1980, le mouvement hip-hop est également importé en Allemagne. Le writing se 
développe notamment sur la façade ouest du mur de Berlin, sous le regard bienveillant des autorités ouest-allemandes. Enfin, la chute du mur qui séparait la ville en deux en passant par son centre ouvre à Berlin de nouveaux espaces vierges de tout usage, au début des années 1990. Les nombreuses marges urbaines ainsi ouvertes sont les lieux privilégiés de la "créativité artistique, esthétique et intellectuelle» berlinoise des années 1990 dont le graffiti est l'un des volets (Grésillon, 2002).

Dans ce contexte, on identifie deux formes de promotion culturelle des graffitis, qui amorcent sa mise en tourisme : la première d'entre elles réside dans le fait de ne pas les effacer. À Paris, la disparition progressive des terrains vagues dans les années 2000 et la mise en place d'un dispositif d'effacement systématique réduit la présence des graffitis dans Paris. À Berlin, malgré la reconstruction de la ville et sa densification progressive, les terrains vagues restent nombreux jusqu'au début des années 2010 et l'absence de dispositif coordonné d'effacement des graffitis rend ces peintures bien plus visibles et plus pérennes qu'à Paris. Le contraste se renforce entre la grande visibilité des graffitis à Berlin et leur relative rareté dans les rues de Paris. Les terrains vagues et les friches des anciens quartiers industriels des capitales deviennent des espaces de tolérance plus officielle des graffitis. On pense notamment au Tacheles à Berlin Mitte, ou aux Frigos dans le $13^{\mathrm{e}}$ arrondissement de Paris. Ces espaces, bien plus nombreux à Berlin qu'à Paris, sont situés dans les quartiers populaires des capitales, ils y attirent les auteurs de graffitis mais aussi un public de plus en plus sensible à ces formes d'expressions murales. La place laissée au développement de la culture " off » (Vivant, 2007) dans les villes est un facteur essentiel de la valorisation des graffitis dans l'espace urbain et de l'attractivité qu'exerce l'espace berlinois dans les années 2000 sur les auteurs et passionnés de graffitis.

Illustration 3 - Graffitis non effacés, rue Dénoyez, dans le $20^{e}$ arrondissement de Paris

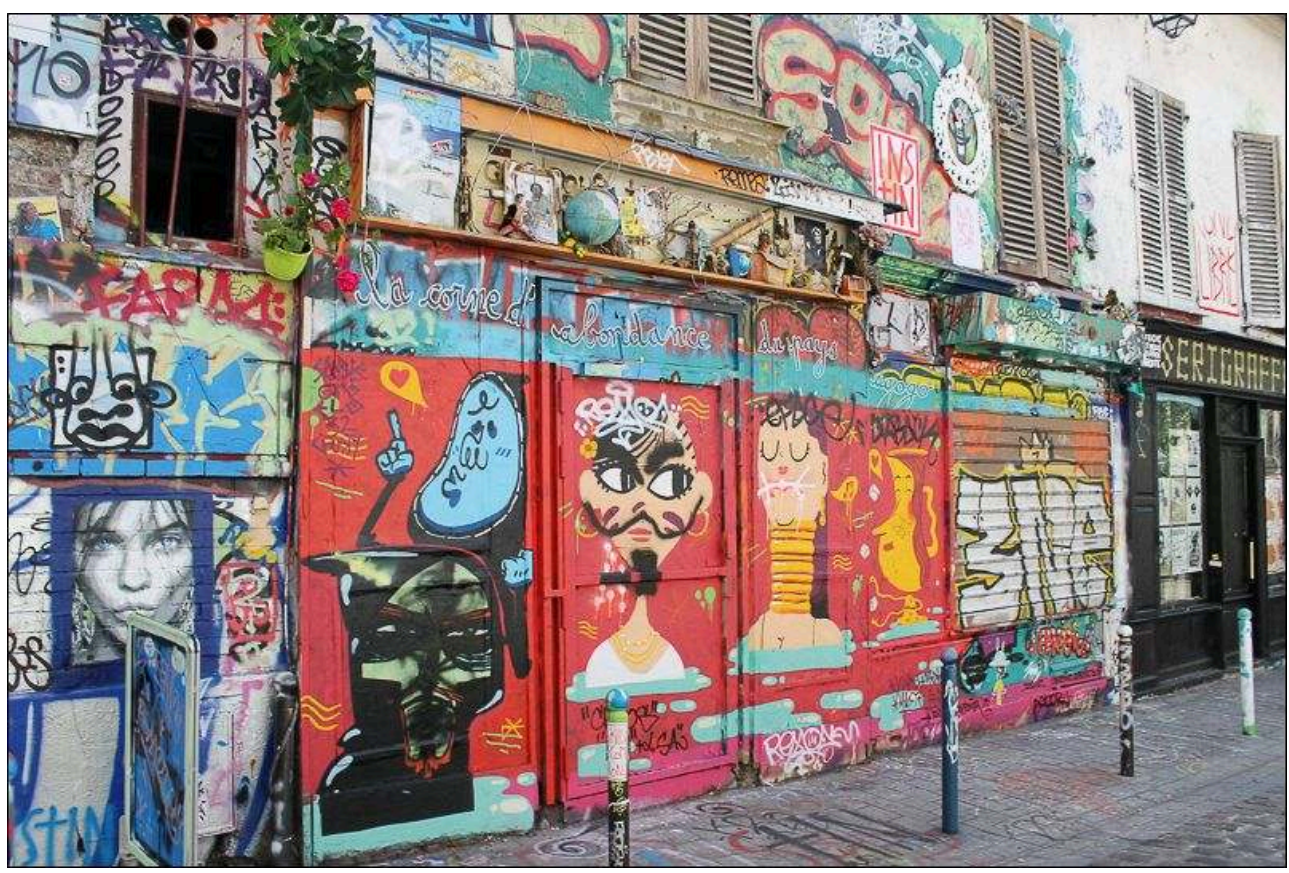

Auteur: J. Vaslin, 2015. 
Illustration 4 - Graffitis non effacés, porche d'entrée de l'Oranienstrasse, arrondissement de Kreuzberg, Berlin

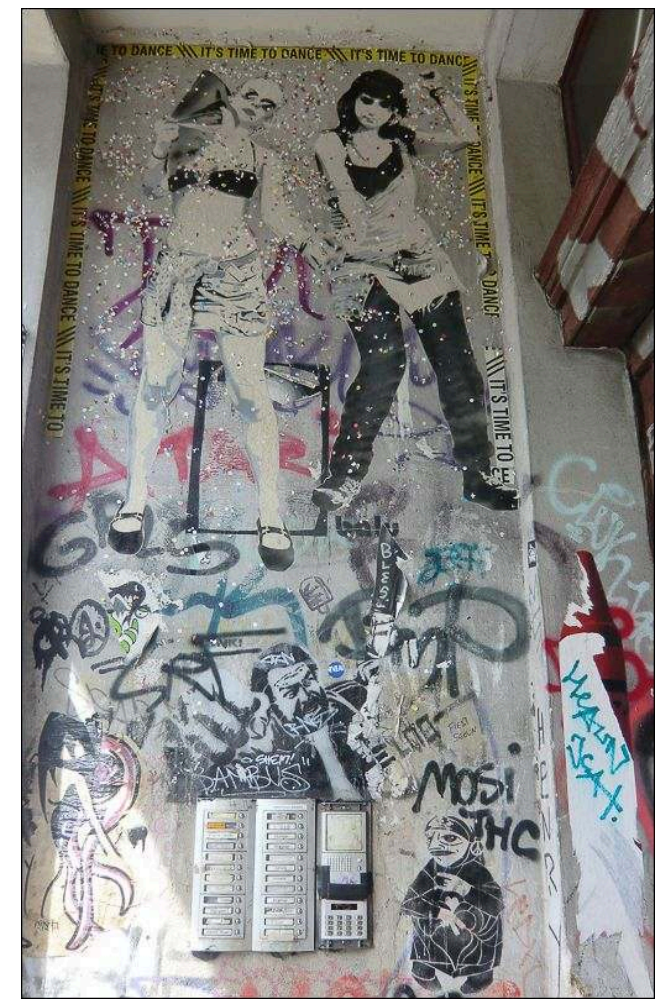

Auteur : J. Vaslin, 2017.

La seconde, plus officielle, consiste à faire entrer le graffiti dans la commande publique d'art. Face à la popularité croissante des lieux où se déploient les graffitis et constatant la professionnalisation de leurs auteurs, les acteurs des politiques culturelles s'impliquent dans la promotion officielle de ces peintures. À Paris comme à Berlin, cette promotion émerge à l'échelle de quelques arrondissements populaires et éloignés des centres touristiques. L'imbrication entre soutien culturel et valorisation territoriale est perceptible dès les premières actions menées par les acteurs publics en faveur des graffitis. Deux exemples sont révélateurs de cette territorialisation des discours. En 2003, le directeur de l'espace d'art Kunstraum Bethanien, institution publique de l'arrondissement Friedrichshain-Kreuzberg, s'associe avec Adrian Nabi, fin connaisseur $\mathrm{du}$ monde du graffiti berlinois, pour produire l'exposition Backjumps. Cette exposition est la première à présenter des graffitis, en tant qu'œuvres, dans un espace d'art contemporain public à Berlin. Ses curateurs la conçoivent en lien étroit avec le lieu d'exposition et le quartier, Kreuzberg, qui l'accueille : «Les travaux de street art [...] m'ont semblé politiques et assez en rapport avec les mouvements sociaux et politiques dans le quartier. [...] Il y avait [à Kreuzberg] beaucoup de groupes politiques engagés [...] qui s'intéressaient à la politique urbaine, la privatisation de l'espace public, etc. [...] Donc mon intention, c'était de relier ces groupes-là, qui n'avaient rien à voir avec le street art, et de leur poser la question "est-ce que le street art n'est pas aussi une forme pour intervenir justement, revendiquer le public pour le public ?" $\gg{ }^{12}$.

La légitimation culturelle du graffiti s'ancre ici dans la promotion d'une identité territoriale fondée sur l'histoire politique du quartier. Reconduit, l'événement Backjumps s'inscrit dans le paysage urbain en invitant les artistes à réaliser des fresques 
monumentales sur les murs de Kreuzberg en 2007. Ces fresques restent, aujourd'hui encore, présentées dans les balades urbaines dédiées au street art.

Illustration 5 - Fresque commandée en 2017 dans le cadre de l'exposition Backjumps, arrondissement de Kreuzberg, Berlin

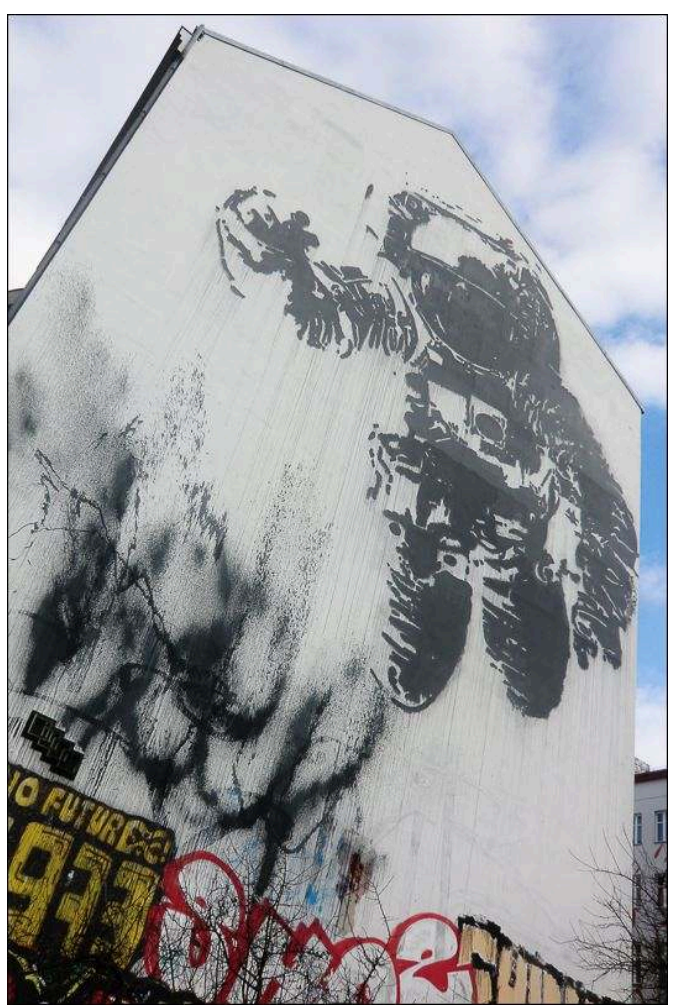

Auteur : J. Vaslin, 2017.

ris, la maire du $20^{\mathrm{e}}$ arrondissement rencontre en 2011 Elise Herszkowisz, fondatrice de l'association Art Azoï, dans le cadre de l'occupation temporaire d'un ancien dépôt de bus par des auteurs de graffitis. Les collaborations entre les deux institutions se multiplient, Art Azoï invite régulièrement des artistes à venir peindre des murs identifiés et mis à disposition par la Mairie du $20^{\mathrm{e}}$ arrondissement. Régulièrement repeints par de nouveaux artistes, ces « murs à programmation » parsèment l'espace du $20^{\mathrm{e}}$ arrondissement et contribuent à le valoriser, autant que les auteurs qui les produisent. Politique culturelle, cette action municipale est également une politique touristique, comme le précise en entretien l'élue d'arrondissement en charge de la culture : "La problématique était, pour l'équipe municipale, que les touristes n'aillent pas voir seulement le cimetière du Père Lachaise [situé dans le $20^{\mathrm{e}}$ ndla]. [...] On n'est pas un arrondissement avec beaucoup de patrimoine dans les grands circuits, donc l'idée a été de penser un développement de territoire à partir d'une originalité, de quelque chose que n'avaient pas les autres $»^{13}$. 
forcément très propre etc. et en même temps, faut pas que ce soit trop le bazar non plus ${ }^{14}$.

Sous l'action conjuguée des projets d'aménagement, de rénovation et grâce au développement d'activités culturelles dans la rue, comme la présence contrôlée des graffitis, le quartier de Belleville est passé aux yeux des acteurs publics d'« infréquentable» à «fréquentable » en quelques années (Chabral, Collet, Giroud, Launay et Ter Minassian, 2016).

Illustration 6 - Mur à programmation dans le $20^{\mathrm{e}}$ arrondissement de Paris

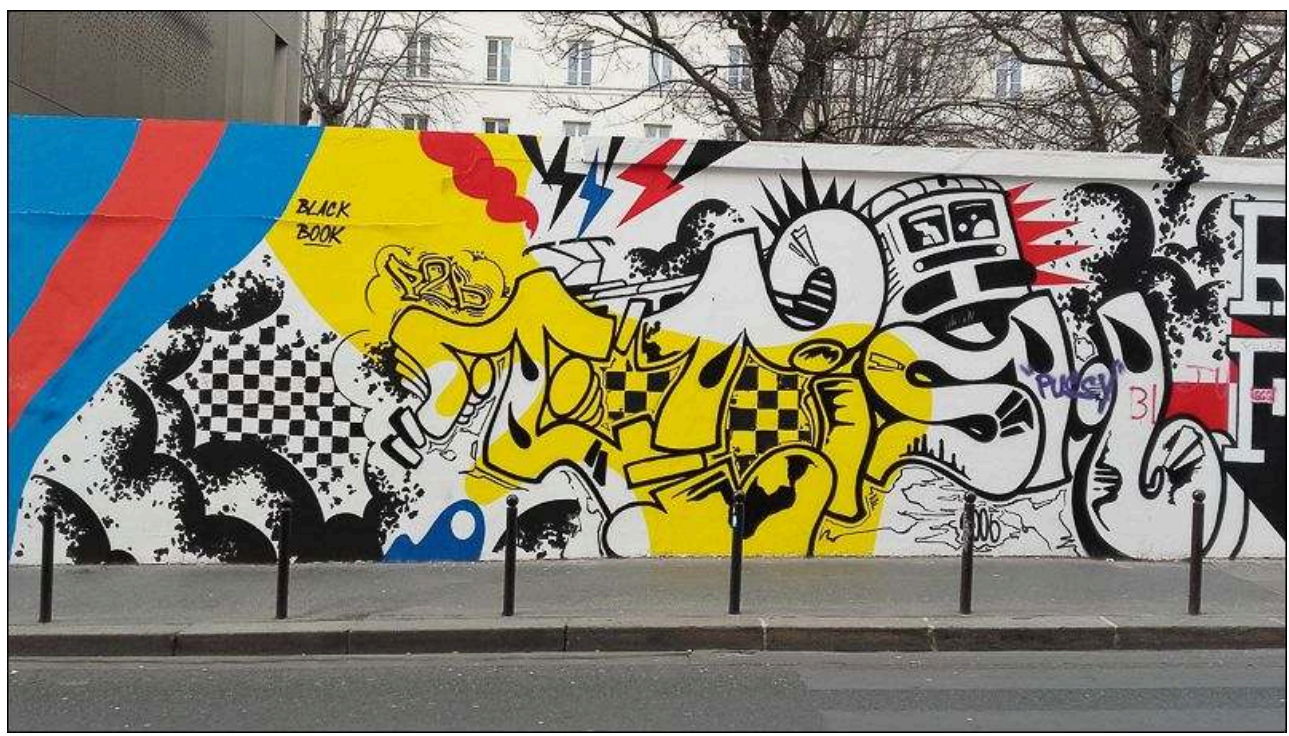

Auteur : J. Vaslin, 2015

À Berlin dans les années 2000 et à Paris dans les années 2010, la commande publique de street art se développe dans le cadre des politiques culturelles de quelques arrondissements populaires. Légitimés dans l'espace public, les graffitis deviennent, aux yeux des autorités urbaines, une pratique esthétique et pacificatrice, propice au renouvellement de l'image du quartier. La promotion culturelle du graffiti entraîne une valorisation du cadre de vie en élaborant "une esthétique particulière, marquée par l'hybridation entre le populaire et le distingué» (Collet, 2015, p.176), pilier de la gentrification et la mise en tourisme des lieux. Cette "esthétisation» des espaces publics renforce l'attractivité touristique de quartiers jusqu'ici restés en marge des circuits touristiques métropolitains et devient le support d'une nouvelle forme de tourisme, « hors des sentiers battus ».

\section{Standardisations des mises en tourisme du street art}

Désignant des pratiques touristiques mues par un attrait pour «les lieux du quotidien ", disposant d'une image au caractère "incontrôlé, inattendu, imprévu ", où se concentrent des activités créatives, le tourisme " hors des sentiers battus » attire des visiteurs issus des catégories moyennes et supérieures dans des espaces urbains qui se démarquent des vitrines touristiques traditionnelles et centrales des métropoles (Gravari-Barbas et Delaplace, 2015). Pour les espaces éloignés des circuits touristiques attractifs, la construction de parcours touristiques hors des sentiers battus constitue un 
élément différenciateur essentiel pour se positionner comme des espaces touristiques particuliers. Dans ce contexte, le street art est devenu incontournable dans l'institutionnalisation d'une offre touristique hors des sentiers battus. Cette offre, de plus en plus standardisée, s'incarne aussi bien dans l'émergence de lieux culturels et festifs que dans des projets d'urbanisme temporaire.

Visible depuis la rue et identifiable grâce aux fresques régulièrement renouvelées sur l'une des façades du lieu, Urban Spree est un lieu culturel situé dans la friche RAW du sud de l'arrondissement Friedrichshain, à Berlin. Créé en 2012 par Pascal Feucher, Urban Spree est à la fois lieu d'exposition, résidence d'artistes, salle de concerts et Biergarten. Après avoir hésité à implanter son projet à Paris, Pascal Feucher installe Urban Spree à Berlin, qu'il qualifie de "ville cool du XXIe siècle ", afin de «tirer parti des 100000 créatifs qui [la] peuplent » (entretien, juin 2013). Misant sur ce qu'il nomme le " label berlinois ", une esthétique " chinée » où « toutes les chaises sont dépareillées ", il s'inspire de l'esthétique des friches culturelles pour proposer une programmation professionnalisée et culturellement légitime à destination d'un public de centre-ville, davantage doté en capitaux économiques et culturels légitimes : «L'idée c'est de tirer le lieu vers le haut, en imposant une programmation plus exigeante et en faisant venir les gens qui refusent de venir [dans le quartier " (entretien avec Pascal Feucher, Berlin, juin 2013).

L'invitation d'artistes street art professionnels est conçue pour attirer des publics éloignés du monde du graffiti dans des lieux culturels qui s'imprègnent d'une esthétique dite "alternative", mais reproduite dans un cadre commercial et institutionnalisé. La programmation du lieu est relayée dans les guides touristiques et les fresques produites à Urban Spree deviennent l'un des arrêts des " tours alternatifs " qui se développent à Berlin depuis le début des années 2000 : l'agence Alternative Berlin, créée en 2006, propose des visites street art, thème de visite largement repris depuis par d'autres prestataires touristiques, en de nombreuses langues. Tout comme les fresques d'Urban Spree, les fresques réalisées dans le cadre de l'exposition Backjumps à Kreuzberg et les graffitis non effacés de l'entrée d'une galerie d'art urbain (Neurotitan) sont régulièrement montrés aux touristes avides de photographier l'esthétique street art de Berlin qu'ils sont venus découvrir clefs en mains dans ces visites. 
Illustration 7 - Façade de Urban Spree, arrondissement de Friedrichshain-Kreuzberg, Kashink

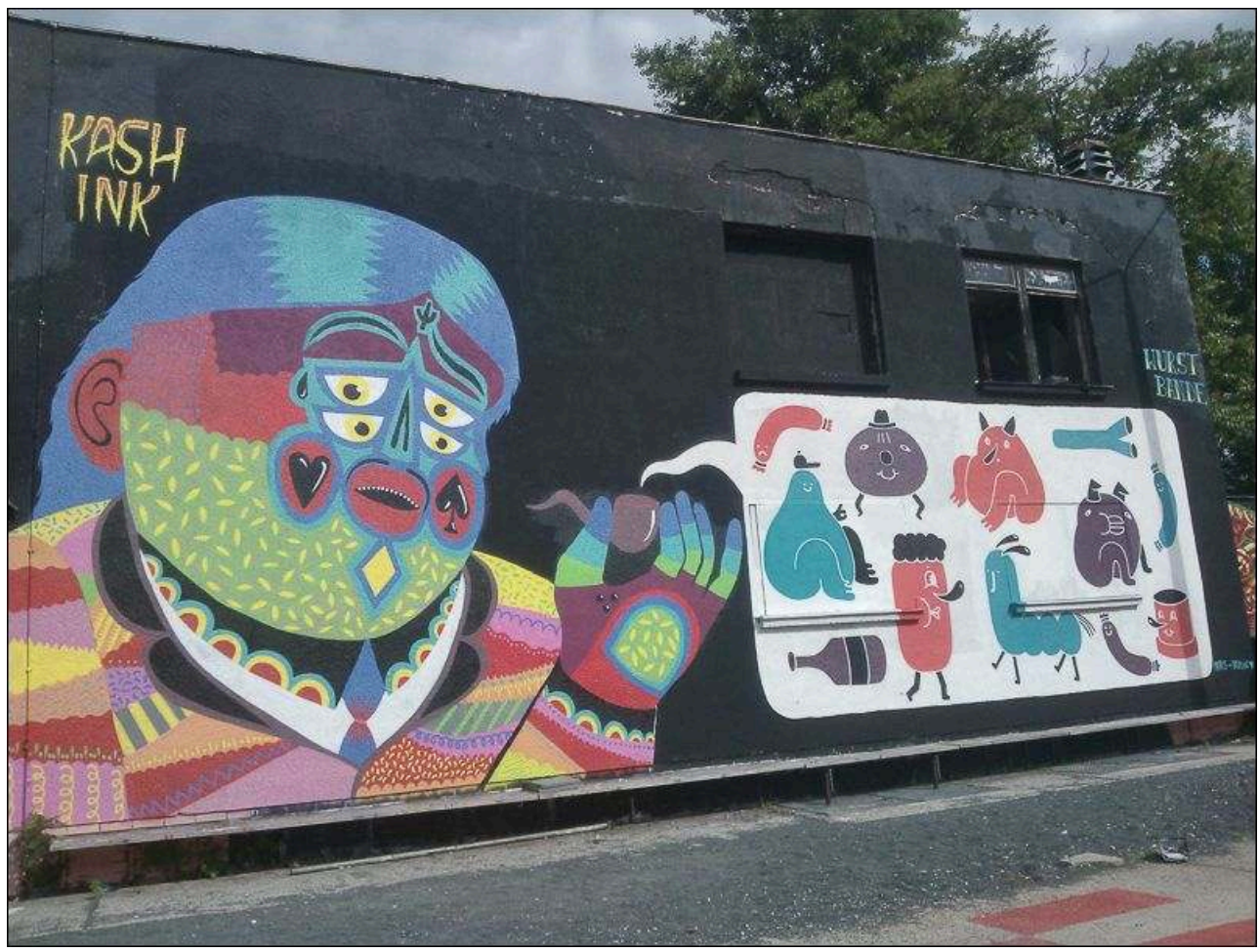

Auteur : J. Vaslin, 2013.

31 À Paris, les visites touristiques de la ville sur le thème du street art se sont également développées ces dernières années. À la différence de Berlin, ces balades urbaines ont été pensées d'emblée par les Mairies d'arrondissement impliquées dans l'encadrement culturel des graffitis. Dès 2009, la Mairie du $20^{\mathrm{e}}$ arrondissement projette de développer autour de l'art urbain un "circuit découverte dans les rues du $20^{e}$ arrondissement ». Mais c'est surtout la Mairie du $13^{\mathrm{e}}$ arrondissement qui, en partenariat avec le galeriste Mehdi Ben Cheikh (galerie Itinerrance), a développé une commande publique de fresque spécialement pensée pour faire émerger un «musée à ciel ouvert» dans l'arrondissement. Renommé street art 13, ce parcours est composé d'une cinquantaine de fresques directement réalisées avec le soutien de la mairie d'arrondissement et des bailleurs, propriétaires des immeubles peints. Ces fresques servent de support à des visites guidées du quartier, par l'entreprise Fresh street art tour ou par l'artiste ThomThom, co-fondateur de l'association MUR dans le $11^{\mathrm{e}}$ arrondissement de Paris. Ces nouveaux acteurs du tourisme parisien organisent également des visites dans le cadre de la jeune programmation street art du comité départemental du tourisme (CDT) de Seine-Saint-Denis. Enfin, un guide touristique spécialement dédié au street art est publié en 2017 (Lombard, 2017). 


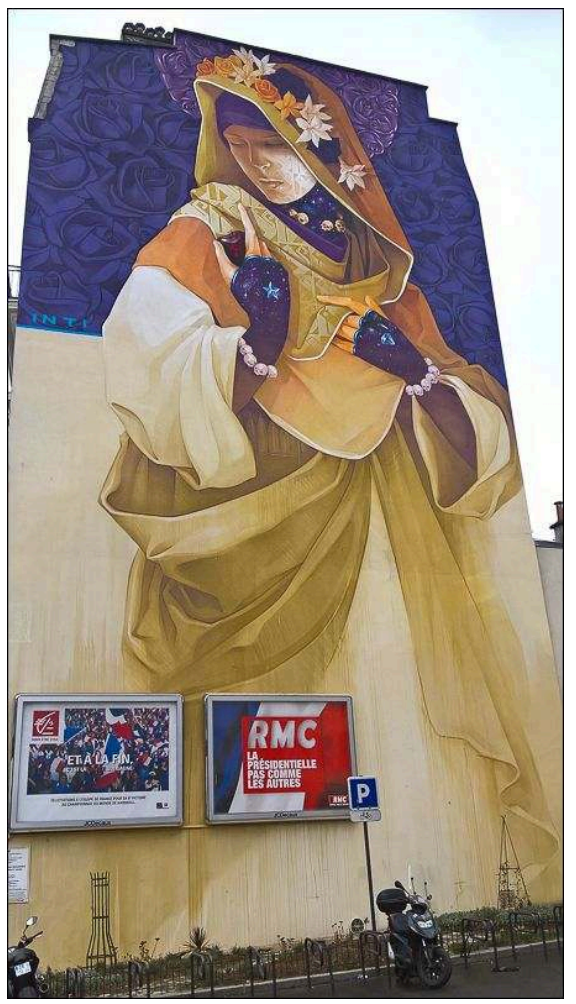

Auteur: J. Vaslin, 2017. mise en tourisme du street art. Sur ce point, les acteurs parisiens ont suivi l'exemple berlinois au milieu des années 2010, s'inspirant des balades urbaines que compte la capitale allemande depuis le milieu des années 2000. Mais ici encore, la préséance du modèle berlinois peut être nuancée, dans la mesure où l'on assiste depuis peu à la reprise, à Berlin, d'un dispositif d'encadrement culturel des graffitis récemment développé à Paris : l'urbanisme temporaire ${ }^{15}$.

Organisée en 2013 par la Mairie du 13 ${ }^{\mathrm{e}}$ arrondissement et le galeriste Mehdi Ben Cheikh, la " Tour 13 » est une exposition temporaire d'artistes internationaux de street art, venus couvrir d'œuvres un immeuble voué à la démolition (Kullmann, 2015). À la suite du succès de cette exposition, plusieurs projets de production temporaire de street art dans des lieux destinés à être détruit ont été menés à Paris. Dans le $12^{\mathrm{e}}$ arrondissement, la Caserne Reuilly a accueilli une exposition dans le cadre du festival «Paris hip-hop» en 2014. Depuis 2015, le projet d'occupation temporaire de l'ancien hôpital Saint-Vincent-de-Paul dans le $14^{\mathrm{e}}$ arrondissement, les "Grands Voisins ", est le théâtre de plusieurs fresques. Au cours de l'hiver 2016-2017, c'est enfin un ancien centre de la Poste qui a accueilli une exposition de street art, le Lab 14, avant d'être entièrement rénové. Ces quelques exemples, saisis parmi tant d'autres, attestent de la multiplication des dispositifs d'urbanisme temporaire à Paris et de la place régulière qu'y tient le street art. Ces projets d'urbanisme deviennent des supports de plus en plus standard de la promotion du street art dans la ville et, par suite, des instruments de développement d'un tourisme hors des sentiers battus, lui aussi fondé sur la dimension en partie éphémère de l'offre. En 2017, à Berlin, l'exposition «The Haus ", organisée dans une ancienne banque en plein arrondissement de 
Charlottenburg, reprend ce principe de promotion temporaire du street art. Se tenant dans l'un des arrondissements les plus aisés de la capitale allemande, cet événement est l'un des rares à quitter les quartiers historiques du graffiti berlinois pour se déployer dans un espace plus central (proche de la Gedächtnis Kirche, haut-lieu touristique berlinois) et moins populaire. De manière générale, l'usage désormais standardisé de dispositifs d'urbanisme temporaire pour encadrer la production de street art favorise le développement ponctuel de cette esthétique dans les quartiers les plus centraux de Paris et Berlin, dans lesquels l'effacement des graffitis spontanés reste par ailleurs le plus intense.

\section{Conclusion}

Malgré des contextes historiques, politiques et urbanistiques très contrastés, la mise en tourisme du street art s'opère, à Paris comme à Berlin, selon des logiques de gouvernement convergentes. Effacé dans les hauts-lieux touristiques des deux capitales européennes, le graffiti est d'abord toléré dans quelques marges urbaines, puis officiellement promu dans quelques arrondissements populaires - sous le label street art. Qu'ils organisent son effacement dans les centres villes ou qu'ils commandent des fresques dans les périphéries des métropoles, les acteurs des politiques urbaines répondent à une même ambition : celle de l'esthétisation des espaces urbains, au nom de l'amélioration du cadre de vie et du développement touristique. Opérée à Berlin dès les années 2000, puis à Paris dans les années 2010, la différenciation des esthétiques urbaines entre le centre-ville et les quartiers périphériques s'appuie sur des dispositifs de plus en plus standardisés de gouvernement des graffitis. D'une capitale à l'autre, les évolutions spatiales du gouvernement du graffiti sont autant de marqueurs, dans l'espace public, de l'institutionnalisation du tourisme hors des sentiers battus dans les métropoles culturelles et touristiques. Voyant leurs productions effacées ou promues en fonction des contextes, les auteurs de graffitis contribuent eux aussi à faire évoluer la place des graffitis dans l'espace public : reste donc ici en suspens, la question de leur rôle dans ces transformations.

\section{BIBLIOGRAPHY}

Austin J., 2001. Taking the train, How graffiti art became an urban crisis in New York City. Columbia University Press, $400 \mathrm{p}$.

Béal V., 2014. "Trendsetting cities" : les modèles à l'heure des politiques urbaines néolibérales. Métropolitiques,: http://www.metropolitiques.eu/Trendsetting-cities-les-modeles-a.html (dernière consultation janvier 2018).

Boichot C., Guinard P., 2013. L'esthétisation des espaces publics à Berlin (Allemagne) et Johannesburg (Afrique du Sud) : l'art pour vendre les espaces publics ou pour rendre les espaces à leurs publics? In Veyrat M. (dir.), Arts et espaces publics. Paris, L'Harmattan, p. 151-177. 
Bocquet D., Laborier P., 2016. Sociologie de Berlin. Paris, La Découverte, 128 p.

Collet A., Launay L., Ter Minassian H., 2016. Une affaire d'image et de représentations. In Chabrol M., Collet A., Giroud M., Launay L., Rousseau M. et Ter Minassian H., Gentrifications. Editions Amsterdam, p. 93-224.

Collet A., 2015. Rester bourgeois. Les quartiers populaires, nouveaux chantiers de la distinction. Paris, La Découverte, $283 \mathrm{p}$.

Cresswell T., 1996. The crucial "where" of graffiti. In In place/out of place: geography, ideology and transgression. Minneapolis, University of Minnesota Press, p. 37.

Ferrell J., 1996. Crimes of style, Urban graffiti and the politics of criminality. Boston, Northeastern University Press, 256 p.

Fleury, A., 2007. Les espaces publics dans les politiques métropolitaines. Réflexions au croisement de trois expériences : de Paris aux quartiers centraux de Berlin et Istanbul. Thèse de doctorat, Université Paris $1,685 \mathrm{p}$.

Fleury A., 2014. La mise en patrimoine des espaces publics. Regards croisés entre Paris et Berlin. In Djament-Tran G. et San Marco P., La métropolisation de la culture et du patrimoine. Éditions Le Manuscrit, p. 221-240.

Gravari-Barbas M., Delaplace M., 2015. Le tourisme urbain "hors des sentiers battus". Téoros Revue de recherches en tourisme [En ligne], $\mathrm{n}^{\circ} 34,1-2$ | 2015, mis en ligne le 14 juin 2016. http:// journals.openedition.org/teoros/2790

Grésillon B., 2008. Ville et création artistique. Pour une autre approche de la géographie culturelle. Annales de géographie, 2008/2, n 660-661, p. 179-198.

Grésillon B., 2002. Berlin, métropole culturelle. Paris, Belin, 352 p.

Guérin F., 2015. L'easyjet-setting de Paris à Berlin. Les enjeux urbains d'une pratique sociale a priori informelle, un type de tourisme d'élite nocturne et festif. Téoros Revue de recherches en tourisme [En ligne], $\mathrm{n}^{\circ} 34,1-2$ | 2015, mis en ligne le 15 mars 2015. http:// journals.openedition.org/teoros/2731

Hassenteufel P., 2008. Sociologie politique :l'action publique. Paris, Armand Colin, 294 p.

Kramer R., 2017. The rise of legal graffiti writing in New York and beyond. Palgrave Pivot, 160 p.

Kullmann C., 2015. De l'exposition de la Tour Paris 13 au concept de musée à ciel ouvert. Le street art au service du projet urbain? Téoros Revue de recherches en tourisme [En ligne], $\mathrm{n}^{\circ} 34,1-2$ | 2015, mis en ligne le 25 avril 2016. http://journals.openedition.org/teoros/2776

Lascoumes P., Le Galès P., 2012. Sociologie de l'action publique. Paris, Armand Colin, 128 p.

Lemoine S., 2012. L'art urbain : du graffiti au street art. Paris, Gallimard, 128 p.

Lombard S., 2017. Guide du Street Art à Paris. Paris, Gallimard, 140 p.

Mairie de Paris, 2017. Stratégie tourisme 2022. Schéma de développement touristique.

Marchand B., 1993. Paris, histoire d'une ville. XIX ${ }^{e}-X X^{e}$ siècle. Paris, Seuil, 443 p.

Payre R., 2008. Ordre politique et gouvernement urbain. Mémoire d'habilitation à diriger des recherches, Université Lyon 2.

Pinçon M., Pinçon-Charlot M., 1989. Dans les beaux quartiers. Paris, Seuil, 256 p.

Pradel B., 2010. Rendez-vous en ville! Urbanisme temporaire et urbanité événementielle : les nouveaux rythmes collectifs. Thèse de doctorat de sociologie, Université Paris-Est, $550 \mathrm{p}$. 
Sanson D., 2014. Berlin. Histoire, promenades, anthologie et dictionnaire. Paris, Robert Lafont, $1152 \mathrm{p}$.

Tilly C., 1984. Les origines du répertoire d'action collective contemporaine en France et en Grande-Bretagne. Vingtième Siècle. Revue d'histoire, n 4, p. 89-108.

Vaslin J., 2017. Esthétique propre. La mise en administration des graffitis à Paris de 1977 à 2017. Thèse de doctorat de science politique, Université de Lyon 2, $661 \mathrm{p}$.

Vivant E., 2007. Les événements off : de la résistance à la mise en scène de la ville créative. Géocarrefour [En ligne], Vol. 82/3 | 2007, mis en ligne le 01 octobre 2010, http:// journals.openedition.org/geocarrefour/2188 ; DOI : 10.4000/geocarrefour.2188;

Weill P.-E., 2014. La consécration du graffiti par le marché de l'art contemporain. Les stratégies complémentaires des intermédiaires. In Lizé W., Naudier D. et Sofio S., Les stratèges de la notoriété. Intermédiaires et consécration dans les univers artistiques. Editions des archives contemporaines, p. 139-164.

Wowereit K., Maire de Berlin, 26 avril 2006, introduction du Congrès Noffiti dont les actes ont été publiés, in Hennig K. (dir)., 2007, Noffiti 2006. Zweiter internationaler Anti-graffiti-Kongress Berlin, Technische Universität Berlin, p. 9.

\section{NOTES}

1. Laurent dans un entretien téléphonique avec Bénédicte et Laurent, fondateurs de l'association Fresh Street Art Tour à Paris, 11 février 2016.

2. Nous désignons ici par "graffiti » l'ensemble des formes d'expression murales réalisées sans autorisation dans l'espace public.

3. Reprise par les professionnels du monde de l'art puis par les pouvoirs publics, l'expression street art désigne ainsi les formes de graffitis sélectionnées et légitimées par ces acteurs, dans des institutions culturelles aussi variées que les galeries, la commande publique, la publicité, la presse ou les guides touristiques. Nous employons donc ici ce terme indigène en italique.

4. Slogan lancé par le maire de Berlin, Klaus Wowereit, en 2003. Voir « Arm, aber sexy“: Wowereit warb in London für Berlin», Tagespiegel, 4/12/2003, http://www.tagesspiegel.de/berlin/armaber-sexy-wowereit-warb-in-london-fuer-berlin/470952.html (dernière consultation le 24/01/2018).

5. Debarbieux, Bernard, «Du haut lieu en général et du Mont Blanc en particulier ", L'espace géographique, 1993, n²2/1, pp.5-13.

6. Source : Eurostat, "Number of nights spent in tourist accommodation establishments in the top 20 EU-28 tourist regions, by NUTS 2 regions, 2015 (million nights spent) », http:// ec.europa.eu/eurostat/statistics-explained/index.php/Tourism_statistics_at_regional_level/

fr\#Nombre_de_nuit.C3.A9es (dernière consultation le 24/01/2018).

7. Ibid.

8. Archives de Paris, Paris, le journal, 20 mai 1992, p. 76.

9. Archives de Paris, Paris, le journal, n92, février 1999.

10. Entretien avec Gabi Dolff Bonekämper, Professeure d'histoire de l'art, TU Berlin, 24 février 2014.

11. Le writing est le nom anglais utilisé pour désigner la pratique du graffiti dans le mouvement hip-hop, un mouvement culturel pluridisciplinaire qui associe une danse, le smurf, le rap et le writing.

12. Entretien avec Stéphane Bauer, directeur de la Kunstraum Bethanien, Kreuzberg, Berlin, février 2014 
13. Entretien téléphonique avec Nathalie Maquoi, adjointe à la Maire du $20^{\mathrm{e}}$ arrondissement en charge de la culture, le 28 mai 2015.

14. Entretien avec Hélène Vicq adjointe à la Maire du $20^{\mathrm{e}}$ arrondissement en charge de l'urbanisme, mairie du 20e arrondissement, place Gambetta, 8 décembre 2014.

15. Le sociologue Benjamin Pradel définit l'urbanisme temporaire comme « [l']action d'organiser l'espace public par des aménagements matériels et symboliques temporaires afin d'amplifier ses usages à court terme et d'accompagner, de valoriser ou d'anticiper des projets urbains à moyen et long terme » (Pradel, 2010).

\section{ABSTRACTS}

Considering that Paris, whose buildings are widely classified as World Heritage by UNESCO, and Berlin, known for being "poor but sexy", are two European capitals with very contrasting images, this article seeks to nuance this opposition by comparing the modalities of the government of the public space in these two cities. Especially, the regulation of graffiti is revealing, in Paris as in Berlin, convergent processes of "aestheticisation" of public space. Erased in high tourist areas, graffiti is tolerated or even promoted in working class areas, where tourism develops off the beaten track. Taken in different timeframes, graffiti governance systems transit from one capital to another, in a game where cultural and touristic developments combine at the service of territorial attractiveness. Considered alternately as an allegory of disorder or as an emblem of alternative tourism, graffiti appears as a very good indicator of these processes.

Partant du constat que Paris, dont le bâti est largement classé au patrimoine mondial de l'Unesco, et Berlin, connue pour être " pauvre mais sexy ", sont deux capitales européennes aux images très contrastées, cet article cherche à nuancer cette opposition en comparant les modalités du gouvernement de l'espace public dans ces deux villes. En particulier, le gouvernement du graffiti est révélateur, à Paris comme à Berlin, de logiques d'esthétisation de l'espace public convergentes. Effacés dans les hauts-lieux touristiques, les graffitis sont tolérés voire promus dans les quartiers populaires périphériques où se développe le tourisme hors des sentiers battus. Pris dans des temporalités différentes, les dispositifs de gouvernement du graffiti transitent d'une capitale à l'autre, dans un jeu où développements culturel et touristique se mêlent, au service de l'attractivité territoriale. Considéré alternativement comme allégorie du désordre ou comme emblème du tourisme alternatif, le graffiti apparaît alors comme un très bon révélateur de ces processus.

\section{INDEX}

Keywords: Graffiti, tourism, urban policy, Paris, Berlin

Mots-clés: graffiti, tourisme, politique urbaine, Paris, Berlin 


\section{AUTHOR}

\section{JULIE VASLIN}

Julie Vaslin, julie.vaslin@sciencespo-lyon.fr, est docteure en science politique, membre du Laboratoire Triangle UMR 5206. Elle a publié récemment :

- Vaslin J., 2018. Discipliner le graffiti. In Lokiss (dir.), Graffiti. 50 ans de collision urbaine. Paris, Hazan (à paraître en octobre 2018)

- Vaslin J., 2018. Le graffiti, pratique esthétique ?, et avec Crettiez X., Les graffitis nationalistes. Étude d'une iconographie contestataire en Corse et au Pays Basque. In Centre des Monuments Nationaux (coord.), Sur le s murs, Histoire(s) de graffitis. Editions du Patrimoine.

- Vaslin J., 2015. Du déviant à l'artistique. Les frontières poreuses de l'étiquetage des graffitis par les pouvoirs publics. In Barbisan L., Bremer M., Marguin S., Kritische Berichte (A), « Kapitalisierung des Marginalen », 2015/3. 